\title{
Gobierno económico, dispositivos de seguridad y normalización biopolítica. Reflexiones en torno a la caja de herramientas foucaulteana
}

Economic government, security dispositives and biopolitical normalization. Reflections about Foucaultean's tool box

Iván Gabriel Dalmau ivandalmau@yahoo.com.ar

http://orcid.org/0000-0003-4045-3488

Universidad Nacional de San Martín/ Universidad de Buenos Aires/ Consejo Nacional de Investigaciones Científicas y Técnicas (Argentina)

\section{Resumen}

El propósito de este artículo es llevar a cabo una lectura minuciosa respecto de la imbricación entre liberalismo, seguridad, biopolítica y la constitución de las modernas ciencias empíricas en los trabajos de Michel Foucault. Por lo tanto, pondremos el foco en la relación entre la gubernamentalidad liberal, la formación de la economía política y la constitución de la población como objeto científico y blanco de intervención política.

Palabras clave: Foucault; liberalismo; seguridad; economía política; población.

\section{Abstract}

The aim of this paper is to make a close reading about the imbrication among liberalism, security, biopolitics and the constitution of the modern empirical sciences in Michel Foucault's 
works. Hence we will focus in the relationship about liberal governmentality, the formation of political economy and the constitution of the population as a scientific objet and as a political intervention target.

Keywords: Foucault; liberalism; security; political economy; population

Luego de la descripción de los dispositivos de seguridad y de los nuevos problemas planteados por la gestión de la población urbana, la exposición se desplaza hacia lo que finalmente, constituye el marco general en el que hay que situar todas sus investigaciones sobre el poder: la historia de la gubernamentalidad (Castro, 2011: 59)

Desde hace poco más de dos décadas, la problematización de la biopolítica elaborada por Michel Foucault ha cobrado una centralidad insoslayable en el seno de las investigaciones y debates del vasto campo, de fronteras difusas, de la denominada Filosofía y Teoría política (Lemke, 2011; Castro, 2014). Esquemáticamente, podría decirse que la proliferación de discursos en torno a dicha categoría oscila entre las indagaciones eruditas, llevadas a cabo por las y los especialistas en filosofía francesa contemporánea, y las apropiaciones críticas sobre las que se configuran vastas líneas de trabajo, desde el pensamiento sociopolítico italiano (Agamben, 1995; Hardt y Negri, 2000; Esposito, 2004; Lazzarato, 2011) y las ciencias sociales anglosajonas (Rose, 2007); hasta amplias líneas de investigación interdisciplinarias desarrolladas en América Latina. En cierto modo, sin ánimos de otorgarle un carácter "demiúrgico" a los vaivenes editoriales, consideramos que no puede desconocerse la relevancia que ha tenido la publicación del primer volumen de la saga Homo Sacer (Agamben, 1995), en el que el filósofo italiano Giorgio Agamben se propone "corregir, o al menos completar, la tesis foucaulteana acerca de labiopolítica"; ni mucho menos puede desatenderse el modo en que la paulatina publicación en formato libro de los cursos dictados por Foucault en el Collège de France ha transformado el "archivo Foucault" (Fontana y Bertani, 1997, Wallenstein, 2013; Raffin, 2015).

Sería pertinente destacar que los cursos dictados por el filósofo durante la segunda mitad de la década del setenta, editados en formato libro entre 1997 y 2004 (Foucault, 1997, 2004a, 2004b) constituyen lo que la crítica ha denominado "cursos biopolíticos". En el primero de ellos, 
dictado en 1976 -que sería, justamente, el primero de los cursos del Collège en publicarse como libro (Foucault, 1997)- la biopolítica es abordada en la última lección del curso, en el marco de la problematización de las mutaciones del discurso de la guerra de razas. Si bien no pueden desconocerse los matices, ni las diferencias de acento, cabría destacar que el modo en que problematiza el "poder sobre la vida", distinguiéndolo del "poder de espada del soberano" es bastante cercano al elaborado en el capítulo final del primer tomo de Histoire de la sexualité (Foucault, 1976), publicado el mismo año en que dictara el curso. Ahora bien, en los cursos siguientes, tomando como punto de partida el análisis de los dispositivos de seguridad (Foucault, 2004a), el foco de problematización se desplaza desde la biopolítica hacia su marco de racionalidad (D’Alessandro, 2011): el liberalismo en tanto forma de racionalización del ejercicio del gobierno en el marco de la soberanía política (Foucault, 2004b).

En ese sentido, no puede pasarse por alto que si en el curso de 1976 Foucault caracterizaba a los dispositivos biopolíticos, que tienen por blanco la regulación de la vida biológica de la población, como modos de "estatización de lo biológico" (1997), en los cursos consecutivos, articulados en torno a la problematización de la formas modernas de gubernamentalidad, propondrá elaborar un análisis que prescinda de tomar como punto de partida "al objeto, la institución y la función" (Foucault, 2004a) y, por el contrario, inscribirá su indagación en el marco de la apuesta teórica y metodológica nominalista que consiste en "suponer que los universales no existen" (Foucault, 2004b). De allí que, en lugar de problematizar al Estado como un "monstruo frío" cuya historia puede trazarse por sí misma, o de convertirlo en un mero reflejo de las transformaciones en las relaciones de producción, propondrá analizar su configuración y sus mutaciones como pliegues y peripecias en la historia de las prácticas gubernamentales. De lo que se trata, entonces, es de llevar a cabo una indagación que prescinda de tomar como punto de partida al sujeto, el Estado y el mercado en tanto términos universales. Apuesta que Foucault caracterizará como "anit-historicista" en la medida en que, en lugar de "pasar los universales por el rallador de la historia", consiste más bien en ponerlos en cuestión y ver qué historia puede hacerse.

Habiendo revisado el ámbito de problematización en que se inscribe el abordaje foucaulteano de la imbricación entre las formas de gobierno económico -el liberalismo y sus inflexiones contemporáneas-, los dispositivos de seguridad y las prácticas de normalización biopolítica, restará decir entonces que dividiremos las líneas que se despliegan a continuación en tres apartados. En el primero, repondremos someramente la genealogía foucaulteana del liberalismo y el neoliberalismo, para enriquecer la presentación del marco en que se despliega su abordaje de los dispositivos de seguridad. La imbricación entre gubernamentalidad liberal, 
normalización aseguradora y formación de saberes respecto de la población constituirá el foco de lectura del siguiente parágrafo. Por último, cerraremos el presente escrito con una breve apartado de reflexión final.

\section{Una historia genealógica (no historicista) de la gubernamentalidad}

Foucault sostiene que con el desarrollo de la economía política se estableció un nuevo principio para la limitación de la racionalidad gubernamental. Mientras que hasta ese momento la ley había funcionado como una limitación externa al gobierno excesivo, el nuevo principio -economía política- era interno a la misma racionalidad gubernamental. Esto significa que el gobierno no tenía que limitarse a sí mismo porque violara la libertad o los derechos básicos de los hombres, sino en vistas del aseguramiento de su propio éxito. (...) En su momento, esto hizo posible juzgarlas como buenas o malas [a las prácticas gubernamentales], no en los términos de algún principio legal o moral, sino en términos de verdad: proposiciones sujetas a la división entre lo verdadero y lo falso. De acuerdo con Foucault, la actividad gubernamental entró, entonces, en un nuevo régimen de verdad

(Oksala, 2013: 57)

Tal como lo señaláramos previamente, en la problematización foucaulteana desarrollada a lo largo de los cursos dictados en el Collège de France durante los ciclos lectivos 1977-1978: Sécurité, Territoire, Population (Foucault, 2004a) y 1978-1979: Naissance de la biopolitique (2004b), el problema de la emergencia de los dispositivos de seguridad es situado dentro del marco de la realización de una "historia de la gubernamentalidad", específicamente en el seno de lo que Foucault llamará, en la última lección del curso de 1977-1978, "gubernamentalidad de los economistas" (2004a), lo cual será retomado en el curso del año siguiente, en el que propondrá "estudiar el liberalismo como marco general de la biopolítica" (2004b). Ahora bien, desde las primeras lecciones del curso de 1977-1878 se advierte la centralidad que para Foucault posee la formación de la economía política para el surgimiento de las formas modernas de gubernamentalidad, como así también para la constitución del objeto población. De hecho, cuando al final del curso contraponga la "gubernamentalidad de los economistas" a la de "los políticos", propia del arte de gobierno según el principio de la Razón de Estado, parte de la estrategia argumentativa consistirá en distinguir y analizar el modo en que fisiócratas y mercantilistas ponen en consideración a la población en el seno de sus problematizaciones. 
Es decir, que Foucault se detendrá en el hecho de que la población pasa de ser un mero dato cuantitativo más, en el seno del análisis "mecánico" respecto de la "fuerza relativa" de los diferentes Estados dentro del marco de la denominada "balanza europea", a cobrar un espesor y una densidad que la tornan un objeto de problematización privilegiado. De "mero dato" para el análisis de las riquezas, devendrá en "realidad espesa" atravesada por dinámicas que, paradójicamente, escapan a una matriz mecánica de problematización, y que se convierte en blanco privilegiado del gobierno económico tal como es problematizado por la naciente economía política de la mano de François Quesnay y la Escuela Fisiocrática francesa. Gobierno económico de la población que se erigirá en torno a la, devenida clásica, fórmula laissez faire-laissez passer y que se articulará estratégicamente a través de los dispositivos de seguridad (Foucault, 2004a).

Sin perder el foco en nuestro trabajo, consideramos pertinente la inclusión de la siguiente caracterización introducida por Foucault, respecto de su propio trabajo, a comienzos del curso de 1978-1979, en donde al brindar un perfil del abordaje de la gubernamentalidad como problema, planteó que:

\footnotetext{
He querido estudiar el arte de gobernar, es decir la manera reflexionada de gobernar mejor y además, al mismo tiempo, la reflexión sobre la mejor manera posible de gobernar. Es decir que intenté abordar la instancia de la reflexión dentro de la práctica de gobierno y acerca de la práctica de gobierno. (...) Intenté determinar la manera a través de la cual se ha establecido el dominio de la práctica del gobierno, sus diferentes objetos, sus reglas generales, sus objetivos de conjunto, con el fin de gobernar de la mejor manera posible. En suma, es (...) el estudio de la racionalización de la práctica gubernamental dentro del ejercicio de la soberanía política (2004b: 4).
}

Retomando la cita que hemos colocado como epígrafe del presente apartado, querríamos remarcar el carácter indisociable del surgimiento del liberalismo como matriz de problematización del ejercicio del gobierno y la constitución de la economía política; ciencia empírica cuya formación se encuentra estrechamente ligada a la emergencia de la problematización del mercado como ámbito de veridicción (Foucault, 2004b). Podría decirse, entonces, que nos encontramos frente a una doble mutación: así como la población deja de ser un "mero dato", y deviene en "realidad densa" frente al ejercicio del poder, el mercado pasa de ser un mero ámbito de jurisdicción, blanco de políticas de controles de precios, por ejemplo, a constituirse en ámbito de producción de la verdad. De este modo, al aparecer "con espesor propio" frente al gobierno, el respeto a los "mecanismos del mercado" emergerá como una 
limitación interna al ejercicio del gobierno, de ahí la "célebre" consigna fisiocrática que hemos mencionado previamente. Puesto que ya no se trata de oponer una limitación externa al ejercicio del gobierno, apelando a cuestiones jurídicas -la violación de un derecho, por ejemplo-, sino que las "verdades del mercado" operan como un filtro intrínseco a la práctica del gobierno, que de no "respetarlas" no comete una injusticia sino una torpeza cuyo efecto será irremediablemente contrario a lo buscado.

Puede decirse, entonces, que las prácticas gubernamentales serán susceptibles de ser analizadas no en términos de justicia e injusticia, sino de adecuación e inadecuación a las verdades inmanentes al mercado, cuyo respeto resulta fundamental para el "éxito" del gobierno. Así, se consolida lo que Foucault denomina como un "gobierno frugal", una suerte de "naturalismo" que hace del mercado una zona vedada para la acción gubernamental; ya que, como lo señaláramos previamente, si el gobierno pretende ser exitoso no puede desconocer ni intentar "espuriamente" violentar sus mecanismos (Foucault, 2004b). De este modo, el liberalismo se consolida en estrecha vinculación con la formación del discurso de la economía política, como una forma de "gobierno esclarecido", cuyo ejercicio no puede desconocer la "realidad espesa" del mercado, a cuyas verdades debe adecuarse.

Ahora bien, la historia de las formas modernas de gubernamentalidad desplegada en estos cursos es completada por Foucault por medio del abordaje genealógico de las inflexiones contemporáneas de la racionalidad política liberal; razón por la cual, le dedicará gran parte del curso correspondiente al ciclo lectivo 1978-1979 al neoliberalismo (2004b), fundamentalmente en sus formas alemana y norteamericana. En dicho contexto, sostuvo Foucault que el neoliberalismo, formado en la Alemania de entreguerras y consolidado en la posguerra, se encuentra ligado fundacionalmente a la Escuela de Friburgo, a la publicación de la revista Ordo y a un conjunto de economistas, sociólogos y juristas, entre quienes se destacan Ludwig von Mises, Friedrich von Hayek, Walter Eucken y Wilhelm Röpke, entre otros. Allí, remarcará el filósofo que el ordoliberalismo -denominación que se le otorga a esta corriente en alusión al título de la citada publicación- se articuló tomando al nazismo como campo de adversidad, problematizándolo como "punto de coalescencia" en el que convergen las distintas formas de dirigismo y planificación económica, como así también las políticas sociales de corte "socialista" (en un sentido amplísimo, lo que implica una radical puesta en cuestión de las políticas de redistribución progresiva del ingreso).

En el marco del citado curso, Foucault remarcará la mutación que había introducido el neoliberalismo respecto del liberalismo clásico, destacando lo inapropiado de una crítica que "denuncie" que los neoliberales pretenden volver al siglo XIX. Básicamente, sostuvo que con la 
Escuela de Friburgo se rompe la ligazón entre liberalismo y laissez-faire, el "naturalismo" al que hemos referido previamente, ya que ellos señalarán que el mercado no es algo dado, una suerte de "dato natural", sino que debe ser constituido activamente. Así, más que gobernar limitando la acción del gobierno en función del "respeto" a los mecanismos del mercado, propondrán que hay que gobernar activamente para producir las condiciones del mercado. De lo que se trata, entonces, es de un activo gobierno del marco que permita inscribir en "la realidad" el mecanismo de la competencia, apuntado hacia la empresarialización de las relaciones sociales. Por lo tanto, si bien cualquier intervención sobre los mecanismos del mercado será impugnada, se alentarán formas de intervención activas sobre las condiciones de posibilidad del mercado. Más que de un retorno al "naturalismo" del siglo XIX, se trata de un "liberalismo sociológico", que en lugar de tomar al mercado como dato y límite, lo problematiza bajo la forma de la competencia en tanto principio formal que debe ser inscripto en "lo real" (Foucault, 2004b).

Ahora bien, siguiendo la lectura foucaulteana no podemos dejar de remarcar que la citada torsión producida por el ordoliberalismo configurado en la Escuela de Friburgo en relación al liberalismo decimonónico, sería profundizada y radicalizada en el marco del desarrollo de la "teoría del capital humano" por parte de la Escuela de Chicago, es decir del neoliberalismo norteamericano, entre cuyos exponentes se destacan Friedrich von Hayek, Milton Friedman y Gary Becker. La citada teoría del capital humano se erige a partir de la problematización del "capital" como "aquello que produce un beneficio", en el contexto de "asignación de recursos limitados hacia fines mutuamente excluyentes"; lo que permite la introducción de un desbloqueo epistemológico al posibilitar la inclusión del "trabajo" como "actividad" dentro del análisis económico (Foucault, 2004b). El "capital humano", en tanto objeto, se constituirá entonces en torno a una serie de capacidades físicas e intelectuales vinculadas a la "productividad" y al savoir-faire atravesadas por la tensión entre lo "innato y lo adquirido".

En el seno de dicha estrategia discursiva, la "grilla de análisis económico" es aplicada a la totalidad de las prácticas sociales, es decir, incluso a aquellos comportamientos considerados "habitualmente" como "no económicos"; desde la educación y las relaciones familiares, hasta la dieta y el acceso a la salud, serán problematizadas en términos de "inversiones en capital humano". En la misma medida en que la grilla económica configura la forma de inteligibilidad de todas las prácticas sociales, se perfila como criterio de intervención gubernamental. Por lo tanto, la contracara de que la racionalidad económica sea problematizada como el modo adecuado y sistemático de responder a las transformaciones de las variables del medio, es que 
el blanco del ejercicio del gobierno se tornará eminentemente gobernable, justamente a través de las intervenciones "esclarecidas" sobre el juego entre dichas variables (Foucault, 2004b).

\section{Saberes, población y normalización: acerca del gobierno económico y sus dispositivos de seguridad}

Tal vez una de las partes más interesantes de Seguridad, territorio, población sea la relectura que hace Foucault de su libro, publicado 12 años antes, Las palabras y las cosas, al final de la clase del 25 de enero de 1978

(Castro Gómez, 2010: 16)

Habiendo explicitado el ámbito de problematización en que se inscribe el abordaje foucaulteano de los dispositivos de seguridad, nos detendremos en este apartado en la imbricación que liga la operatoria de dichos dispositivos normalizadores con la racionalidad económica de gobierno. Tal como lo señaláramos previamente, recordamos que dichas problemáticas se despliegan en el marco del sendero abierto por las indagaciones del filósofo respecto de la biopolítica. En sintonía con la cita que hemos colocado como epígrafe, revisaremos el modo en que Foucault problematiza la imbricación entre gobierno económico, dispositivos de seguridad y saberes acerca de "la vida, el trabajo, el lenguaje y lo humano". En ese sentido, sería pertinente destacar que en las primeras clases de Sécurité, Territoire, Population (2004a), el pensador francés se ocupó de desbrozar las diferentes maneras en que desde la perspectiva de la soberanía, la disciplina y la seguridad se constituyó como problema a "la ciudad", "la escasez" y "las epidemias". Sin embargo, buscando disipar cualquier tipo de lectura de carácter "etapista", el filósofo destacó que no habría que entender dicha distinción en términos de "eras" en las que los distintos modos de ejercicio del poder se sucederían, reemplazando a los precedentes; según sus propios términos:

No hay era de lo legal, era de lo disciplinario, era de la seguridad. No hay mecanismos de seguridad que tomen el lugar de los mecanismos disciplinarios, los cuales habrían tomado el lugar de los mecanismos jurídico-legales. De hecho, tenemos una serie de edificios complejos entre los cuales lo que va a cambiar, por supuesto, son las técnicas que se van a perfeccionar, o en todo caso complicar, pero sobre todo lo que va a cambiar es la dominante o, más exactamente, el sistema de correlación entre los mecanismos jurídicolegales, los mecanismos disciplinarios y los mecanismos de seguridad (2004a: 10). 
No podemos dejar de mencionar que, en el marco del citado curso, Foucault problematiza el surgimiento de la normalización biopolítica, articulada a través de los dispositivos de seguridad, como un acontecimiento que no es susceptible de ser desligado de la emergencia de ciertos problemas de carácter científico-político, en torno a los cuales la población se constituyó como sujeto-objeto de manera correlativa al desbloqueo de las artes de gobierno. Estas, justamente, se reconfigurarán en la modernidad europea al tomar a la población como blanco de intervención y objetivo de su práctica. Retomando lo señalado en el apartado precedente, consideramos fundamental remarcar que la mutación del modo en que se constituye la población como objeto de saber y blanco de intervención política, trajo aparejada una profunda transformación epistemológica y ontológico-política. En el seno de dicho acontecimiento, la población se re-constituirá como objeto que, por su espesor ontológico escapa a las mallas reglamentarias. En cierto modo, podría decirse que la población aparece frente al gobierno como un objeto en parte inasible, en la medida en que el dinamismo de las variables que la atraviesan escapa tanto a los dispositivos legales, como a las reglamentaciones disciplinarias. De alguna manera, podría decirse que "lo poblacional" se encuentra atravesado por normas cuya operatoria poseen una plasticidad, una tendencia al desajuste y al reequilibrio, que desbordan no sólo la noción jurídica de "ley", sino también la noción "físico-mecánica", habida cuenta de que, por ejemplo, son inherentes a la operatoria de las "leyes económicas" y de las normas biológicas, la crisis, el desajuste, el desequilibrio, la patología. Ahora bien, ¿no es esa misma plasticidad la que las torna eminentemente gobernables? Cabe recordar que en el marco del curso en cuestión, Foucault sostuvo que si bien las mutaciones epistemológicas de los saberes respecto del cosmos no tienen incidencia sobre las dinámicas cosmológicas, no puede decirse lo mismo acerca de los saberes respecto de, por ejemplo, la política (2004a). Nos permitimos plantear que, en la medida en que se entrelazan con el ejercicio de un "gobierno esclarecido", y que su formación se encuentra ligada a la constitución de un objeto poroso como lo es la población (emplazada en un determinado medio), los saberes acerca de "la vida, el trabajo, el lenguaje y lo humano" no carecen de efectos sobre "lo real". En términos del reconocido especialista alemán Thomas Lemke:

De acuerdo con Foucault, la biopolítica no complementa las competencias y estructuras políticas tradicionales a través de nuevos dominios y problemas. No produce una extensión de la política sino que más bien transforma su núcleo, reformula los conceptos de la soberanía política y los subyuga a nuevas formas de saber político. La biopolítica se apoya 
en una constelación en la cual las ciencias humanas y naturales, y los conceptos normativos que de ellas emergen, estructuran la acción política y determinan sus objetivos (2011: 33).

Llegados a este punto, en línea con la cita del especialista colombiano Santiago Castro Gómez que hemos colocado como epígrafe, consideramos pertinente detenernos en la clase del 25 de enero de 1978, correspondiente al curso Sécurité, Territoire, Population. Allí, al ocuparse de la citada distinción entre el modo de ejercicio del poder propio de las tecnologías de seguridad frente a los dispositivos disciplinarios y a la lógica de la soberanía, Foucault remarcó -en torno a la contraposición entre "normación" y "normalización"- la especificidad de la manera en que desde las perspectivas disciplinarias y de seguridad se ligan "norma" y "normalidad" respecto del objeto que se erige como problema frente al ejercicio del poder. Dentro de dicho análisis, destacará que las tecnologías de seguridad ponen en juego la "naturalidad del objeto mismo" que es blanco del ejercicio del gobierno, configurándose la "norma" de manera inmanente al juego de las "normalidades diferenciales" que lo atraviesan. Al respecto, no podemos dejar de hacernos eco del modo en que el reconocido filósofo francés Philippe Sabot caracterizó dicha distinción introducida por Foucault en torno al problema de las normas:

(...) A finales de los años setenta Foucault realiza un desplazamiento con respecto a su análisis que pasa a través de una distinción interesante entre dos modos de normalización. (...) Foucault completa el análisis de la operación normación-conformación por referencia a la dimensión de otras "técnicas de normalización" que reenvían, en realidad, a otra lógica normativa, inmanente y relativa a los denominados "dispositivos de seguridad" (Sabot, 2016).

En la misma dirección, la investigadora alemana Maria Muhle sostuvo que:

Los dispositivos de seguridad dejan libre curso a los fenómenos vitales pero se implantan sobre su dinámica: crean las condiciones, el medio, en las cuales la vida se puede autorregular libremente (...). En este sentido debe ser comprendida la definición foucaulteana de la biopolítica como poder positivo sobre la vida: es un poder que se apoya sobre la productividad interna de la vida y se inscribe en su normatividad con el fin de dominar a su objeto de manera más eficaz (2012: 194-195). 
Las citas que acabamos de incluir dan cuenta del modo en que, en la perspectiva foucaulteana, se produce una profunda imbricación entre la configuración de las formas de gobierno económico, indisociable de la formación de determinadas ciencias empíricas que permiten la constitución de una forma de "gobierno esclarecido", y el surgimiento de los dispositivos de seguridad. Dispositivos cuya operatoria y "eficacia" se erigen a partir del modo en que "administran el medio" en que se encuentra emplazada la población, apoyándose sobre la "libre oscilación" de sus variables. Por otra parte, tal como lo señaláramos previamente, sería pertinente destacar que la población emergerá como un sujeto-objeto a gobernar que, por su espesor y "naturalidad", escapa a las "mallas reglamentarias" configuradas a partir de la "voluntad soberana" (Nalli, 2014). Como sostuviera el investigador argentino Manuel Mauer:

\begin{abstract}
Así, la misma naturalidad que torna refractaria a la población con respecto a los decretos del soberano, la vuelve, al mismo tiempo, accesible a técnicas de transformación, bajo la condición de que ellas sean esclarecidas, reflexionadas y calculadas: para actuar sobre ella deberán, en efecto, hacer jugar una serie de factores que se encuentran aparentemente alejados de la población (por ejemplo, los impuestos, la infraestructura, los flujos monetarios), pero a los cuales se encuentra ligados. (...) La población no es, entonces, ni una colección de sujetos jurídicos relacionados con una voluntad soberana, ni un conjunto de cuerpos individuales atrapados por instituciones disciplinarias. Ella reenvía, más bien, a una masa que se inserta dentro del régimen general de los seres vivientes y ofrece, por el mismo motivo, una superficie de agarre para transformaciones reflexivas y calculadas (2015: 46).
\end{abstract}

En sintonía con lo antedicho, Michel Foucault planteó que en el marco del surgimiento de las tecnologías de seguridad, se produjo una profunda imbricación entre la visibilización de ciertos problemas políticos y la constitución de determinados objetos de saber. En sus propios términos:

(...) Un juego incesante entre las técnicas de poder y su objeto recortó poco a poco en lo real y como campo de realidad a la población y sus fenómenos específicos. Y es a partir de la constitución de la población como correlato de las técnicas de poder que se pudieron abrir toda una serie de dominios de objetos para saberes posibles. En revancha, es porque esos saberes recortaron sin cesar nuevos objetos que la población pudo constituirse, prolongarse y mantenerse como correlato privilegiado de los mecanismos modernos de poder (Foucault, 2004: 80-81). 
La formación del discurso de la economía política, la biología y la filología, en cuyo plexo se configuraron las ciencias humanas que Foucault había abordado doce años antes en Les mots et les choses (1966), será puesta en relación con el acontecimiento de emergencia de determinadas problematizaciones científico-políticas que atravesaron el surgimiento de las formas de gobierno económico y sus dispositivos de seguridad. Puede plantearse entonces que el acontecimiento de emergencia de los dispositivos de seguridad, en cuyo seno se configuró la biopolítica, en tanto forma de administración y regulación de la vida biológica de la población, resulta indisociable de la constitución de ciertos objetos de saber que operaron a su vez como blancos de intervención política de la naciente tecnología de poder; imbricación indisociable para Foucault, en tanto se trata del surgimiento de una forma de "gobierno esclarecido". En dicho contexto, Foucault sostuvo que el hombre, objeto de las ciencias humanas y de la reflexión filosófica humanista, debe ser considerado como una "figura de la población".

\section{Palabras finales}

Aunque resulta evidente que el carácter y el uso crítico de las categorías de ningún modo pueden orientarse a la preservación de aquello que critican, de todas formas, M. Foucault (...) se ocupó de subrayar el cariz político y transformador que atribuía a la actividad intelectual. (...) Su interpretación de la crítica como apelación a no ser más gobernados de la misma manera no puede ser sino leída en línea directa con el problema de la gubernamentalidad que genera el liberalismo

(López, 2012: 51)

A lo largo del presente artículo hemos revisado el modo en que, en el marco de los denominados "cursos sobre biopolítica" dictados por Michel Foucault durante la segunda mitad de la década del setenta en el Collège de France, el filósofo orienta sus indagaciones hacia la realización de una genealogía de las formas modernas de gubernamentalidad. En dicho contexto, colocamos el foco de lectura en el modo en que problematiza la imbricación entre el surgimiento del liberalismo, en tanto modo económico de racionalizar el ejercicio del gobierno, la formación de la economía política, que habilita a la constitución de una forma "esclarecida de gobernar", y la emergencia de los dispositivos de seguridad. Dispositivos que al apoyarse en la "naturalidad" de la población y sus múltiples relaciones con el medio en que se encuentra emplazada, operan a través de la administración de la libertad de "oscilación" de las variables que atraviesan, en su porosidad, a dicho objeto. Objeto cuya "densidad porosa" es 
problematizada por las ciencias empíricas; es decir, no sólo la economía política sino además la filología y la biología, como así también por las ciencias humanas. En la medida en que las prácticas de regulación biopolítica se articulan por medio de la operatoria de los dispositivos de seguridad, configurados en el marco de la denominada "gubernamentalidad de los economistas", puede captarse por qué Foucault se desplaza de la biopolítica hacia el liberalismo, en tanto marco de racionalidad en que la misma pudo formarse.

Ahora bien, no queríamos dejar de cerrar este escrito sin remarcar, en línea con la cita de la reconocida especialista argentina Cristina López, la tonalidad crítica que vertebra los trabajos foucaulteanos, y que consideramos fundamental, en la medida en que encontramos allí una caja de herramientas para la problematización de nuestra actualidad. En cierto modo, podría decirse que si las formas de gobierno neoliberal emergieron como fruto de una torsión, mas no ruptura, respecto de la racionalidad liberal, la cantera de trabajo que el "archivo Foucault" posibilita, radica ni más ni menos en el modo en que problematiza aquellas formas de ejercicio del gobierno que se configuran por medio de la "administración de la libertad de los gobernados". Frente a estas prácticas gubernamentales, el ejercicio de la filosofía y de la reflexión teórico-política se configura en torno a la posibilidad de articular un decir veraz (Foucault, 2009) que desafíe al poder. ¿Cómo articular una forma de vida configurada en torno al coraje de la verdad, que contribuya al ejercicio de la crítica en tanto "arte de la insubordinación voluntaria" (Foucault, 1990)? Intentando remarcar la tonalidad política de estas indagaciones, retomamos una distinción introducida por Foucault en el citado curso de 1978 (2004a) y nos preguntamos: ¿frente a las formas de gobierno que buscan "administrar" una población y "gestionar" las opiniones del público, cómo contribuir a la emergencia desafiante del pueblo? Pueblo que, en su manifestarse frente al poder, ponga en cuestión sus formas de racionalización al articular una verdad-otra (Foucault, 2009).

\section{Bibliografía}

AA.VV. (2012). Epistemology and History. From Bachelard and Canguilhem to Today's History of Science. Alemania: Max Planck Institutfor the History of Science.

Agamben, G. (1995). Homo Sacer. II potere sovrano e la nuda vita, Torino, Einaudi.

Castro, E. (2011). Lecturas foucaulteanas. Una historia conceptual de la biopolítica. Buenos Aires: UNIPE, Editorial Universitaria. 
Castro, E. (2014). Los malentendidos de la biopolítica: Foucault, Agamben, Derrida. Quadranti. Rivista Internazionale di Filosofia Contemporanea, Salerno, Università di Salerno, 2(2), pp. 109-124.

Castro Gómez, S. (2010). Historia de la gubernamentalidad. Razón de Estado, liberalismo y neoliberalismo en Michel Foucault. Bogotá: Siglo del Hombre Editores.

D’Alessandro, D. (2011). Filosofie della biopolitca. Figura e problemi, Salerno (Tesi di Dotorato in Etica e Filosofia politco-giuridica Ciclo X). Univesità Degli Studi Salerno, Italia.

Esposito, R. (2004). Bíos. Biopolitica e filosofia. Torino: Einaudi.

Fontana, A. y Bertani, M. (1997). Situation du Cours. En Foucault, M. «ll faut défendre la société». Cours au Collège de France, 1976. Paris: Éditions Gallimard.

Foucault, M. (1966). Les mots et les choses. Une archéologie des sciences humaines. Paris: Gallimard.

Foucault, M. (1976). Histoire de la sexualité 1. La volonté de savoir. Paris: Éditions Gallimard.

Foucault, M. (1990). Qu’est-ce que la Critique? (Critique et Aufklrärung). Bulletin de la Société française de Philosophie, Paris, 84(2), pp. 35-63.

Foucault, M. (1997). “ll faut défendre la société». Cours au Collège de France, 1976. Paris: Éditions Gallimard.

Foucault, M. (2004a). Sécurité, Territoire, Population. Cours au Collège de France. 1977-1978. Paris: Éditions Gallimard.

Foucault, M. (2004b). Naissance de la biopolitique. Cours au Collège de France. 1978-1979. Paris: Éditions Gallimard.

Foucault, M. (2009). Le courage de la vérité. Le gouvernement de soi et des autres II. Cours au Collège de France. 1983-1984. Paris: Éditions Gallimard.

Hardt, M. y Negri, A. (2000). Empire. United States of America: Harvard UniversityPress.

Lazzarato, M. (2011). La fabrique de l'homme endetté, essai sur la condition néolibérale. Paris: Éditions Amsterdam.

Lemke, Th. (2011). Biopolitics. An advanced introduction. New York: New York University Press.

López, C. (2012). De los usos y abusos de la biopolítica foucaulteana. En Raffin, M. y Podestá, B. Problemas y Debates de la Tradición y la Actualidad de la Filosofía Política. San Juan: EFFHA.

Mauer, M. (2015). Foucault et le problème de la vie. Paris: Publications de la Sorbonne. 
Muhle, M. (2012). Histoire(s) de la vie de Canguilhem a Foucaul. En AA.VV. Epistemology and History. From Bachelard and Canguilhem to Today's History of Science. Max Planck Institut for the History of Science, pp. 187-196.

Nalli, M. (2014). The Normative Immanence of Life and Death in Foucauldian Analysis of Biopolitics. Materiali Foucaultiani, 3(5), pp. 196-218.

Oksala, J. (2013). Neoliberalism and Biopolitical Governmentality. En Nilsson, J. y Wallenstein, S. O. (Eds.). Foucault, Biopolitics and Governmentality (pp. 53-72). Stockholm: Södertörn University The Library.

Raffin, M. (2015). La verdad y las formas políticas: la lectura temprana de la tragedia de Edipo en Michel Foucault. Anacronismo e Irrupción. Revista de Teoría y Filosofía Política Clásica y Moderna, 5(8).

Rose, N. (2007). The Politics of Life Itself. Biomedicine, Power and Subjectivity in the TwentyFirst Century. New Jersey: Princeton University Press.

Sabot, Ph. (2016). De Foucault à Macherey, penser les normes. Methodos. Savoirs et textes. doi: 10.4000 /methodos. 4652

Wallenstein, S. O. (2013). Introduction: Foucault, Biopolitics, and Governmentality. En Nisson, J. y Wallenstein, S. O. (Eds.). Foucault, Biopolitics and Governmentality. Stockholm: Södertörn University The Library. 